\title{
Health insurance status and outcomes in children, adolescents, and young adults: a systematic review and meta-analysis
}

\author{
Congyang Huang ${ }^{1,2 \oplus}$, Hanshan $\mathrm{Liu}^{3 \oplus}$, Honglian $\mathrm{Hu}^{4 \oplus}$, $\mathrm{Li} \mathrm{Jia}^{4 \oplus}$, Suyun $\mathrm{Hu}^{5 \oplus}$ \\ ${ }^{1}$ Institute of Economics, Shanghai Academy of Social Sciences, Shanghai, PR China; ${ }^{2}$ The Bureau of Jiangyin Human Resources and \\ Social Security, Jiangyin, Jiangsu, PR China; ${ }^{3}$ Second Department of Internal Medicine, Jiangsu Provincial Corps Hospital of Chinese \\ People's Armed Police Forces, Yangzhou, Jiangsu, PR China; ${ }^{4}$ Department of Nephrology, Ningbo Yinzhou No.2 Hospital, Ningbo, \\ Zhejiang, PR China; ${ }^{5}$ Institute for Urban and Population Development, Shanghai Academy of Social Sciences, Shanghai, PR China.
}

\begin{abstract}
Background. The impacts of health insurance status on survival outcomes in children, adolescents, and young adults (aged 0-39 years) with malignant tumors have not been addressed in depth. The present study aimed to identify significant relationships of health insurance condition with overall survival or all-cause mortality among children (age 0-14 years) and adolescents and young adults (AYAs, age 15-39 years) with malignant tumors.

Methods. PubMed, Wiley Cochrane Central Register of Controlled Trials, Econlit, CINAHL, Web of Knowledge, PsychInfo, Business Source Premier, ProQuest Dissertation \& Theses Database, and SCOPUS were systematically searched from inception to February 29, 2020 with no language restriction. All related articles comparing the effect of health insurance status on the risk of overall survival and the risk of all-cause mortality in malignant conditions affecting children and AYAs were identified. Pooled risk ratios (RRs) and 95\% confidence intervals (CIs) were computed using a random- or fixed-effect model as per the heterogeneity evaluated using Cochran's $\mathrm{Q}$ and $\mathrm{I}^{2}$ statistics.
\end{abstract}

Results. Fourteen studies including 149,680 individuals were selected for this meta-analysis. The pooled RR for all-cause mortality with insurance versus without insurance was 0.78 (95\%CI, $\left.0.71-0.86 ; \mathrm{I}^{2}=33.7 \%\right)$. Among the insurance types, patients with private insurance presented with a lower all-cause mortality (RR 0.70, 95\% CI $0.60-0.82)$, with considerable heterogeneity $\left(I^{2}=83.3 \%\right)$.

Conclusions. The findings of this review suggest that a lack of or insufficient insurance is related to all-cause mortality of AYAs with malignant cancers. Strategies aimed at identifying causality and reducing disparities are warranted.

Key words: children, adolescents and young adults, malignancy, health insurance, survival analysis.

The survival of malignancy-affected children ${ }^{1}$ and adolescents and young adults (AYAs, aged 15-39 years) ${ }^{2}$ has largely improved in the past 50 decades due to the remarkable progress in medicine, including diagnostics, pharmacology, combined treatments and techniques. Leading causes of death among

$凶$ Suyun $\mathrm{Hu}$

qingzxyry@sina.com

Received 9th February 2021, revised 9th March 2021, accepted 24th March 2021. all mortality reasons in this age group vary as a function of age, sex, and Socio-demographic Index (SDI) status, a composite indicator of development status generated for the Global Burden of Diseases, Injuries, and Risk Factors (GBD). ${ }^{3}$ A data from the 2004 GBD $^{4}$ found that traffic accidents were the largest cause in both sexes, and maternal conditions were a leading cause of female deaths. Nevertheless, in data from the 2013 GBD with age range from 1 to 24 years, it was found that neoplasms had become the major cause of death in 1-9 year olds of both sexes, and in 10- to 24-year-old females in 
Malaysia ${ }^{5}$, although the major cause of death in 10- to 24-year-old males remained road traffic injuries. Meanwhile, malignancy remains the dominant cause of disease-linked death among these age groups in most studies..$^{6-8}$ These populations do not benefit equally from these advances, owing to discrepancies in the age of clinical occurrence, malignancy type, disease stage (in solid tumor), anatomical position, geological region, or variations within regions. Discrepancies are particularly evident in resource-restricted settings, but also apply to socioeconomic groups in developed countries, although to a lesser but detectable degree. ${ }^{9}$ In undeveloped countries, survival rates of cancerbearing AYAs and children are dismal and lower than $10 \% .^{10}$ In high-income countries, the survival rates of AYAs with cancer have improved, while the survival of younger pediatric patients and older adult patients have deteriorated because of inaccessibility to medical care and health insurance, as well as disparities in race/ethnicity and neighborhood socioeconomic status (SES). ${ }^{11}$ American AYAs are the least likely to be covered by health insurance, and they tend to choose no insurance or public insurance. ${ }^{12}$ Recent research implies that insurance deficiency is positively related to delayed diagnosis and later stage (and less treatable) disease in children and AYAs with cancer. ${ }^{13}$ Insured young adults tend to undergo definitive cancer therapy and are therefore, less likely to die. ${ }^{13}$ Additionally, lower neighborhood SES, minority race/ethnicity, public or deficient insurance, and other sociodemographic factors are all related to a greater risk of death ${ }^{14}$ among AYAs, in addition to delayed diagnoses and undertreatment. $^{13}$

However, the effect of health insurance on the risk of mortality in cancer-bearing AYAs (age 1539 years) and children (age $0-14$ years) has not been clearly described because of inconsistent findings. ${ }^{15,16}$ In particular, some undetected confounders may have been neglected by model adjustment and contributed to bias. Given these concerns, we aimed to verify these findings through a systematic review.

\section{Material and Methods}

\section{Search scheme and inclusion criteria}

This study adheres to the PRISMA statement standards of quality for reporting systematic reviews. Two independent investigators searched PubMed, Wiley Cochrane Central Register of Controlled Trials, Econlit, CINAHL, Web of Knowledge, PsychInfo, Business Source Premier, ProQuest Dissertation \& Theses Database, and SCOPUS with starting and ending date from inception to February 29, 2020 and with no language restriction. The combinations of terms searched included: 'insurance', 'Medicaid', 'Medicare', or 'cooperative medical scheme' and 'infant', 'child"', 'adolescent', 'youth"', 'puberty', 'prepuberty*', 'pediatric ${ }^{* \prime}$, or 'paediatric ${ }^{* \prime}$ and 'cancer', 'oncolog*', 'neoplas", 'carcinom*', 'tumor", 'malignan*', 'tumour ${ }^{* \prime}$, 'leukemi ${ }^{* \prime}$, 'lymphom*', 'sarcom*', 'osteosarcoma', 'nephroblastom*', 'neuroblastoma', 'rhabdomyosarcoma', 'teratom', 'hepatom*', 'hepatoblastom"', 'medulloblastom", 'retinoblastom*', 'meningiom*', or 'gliom*' and 'mortality', 'mortalit', or 'survival'. The titles and abstracts of papers as-searched were reviewed. The search scheme was elaborated in Supplementary Information. The references of these papers were hand-searched using the snow-ball technique to ensure no potential articles were missed. Disagreements were resolved through mutual consensus. When detailed information needed for the analysis was unavailable, the original authors were contacted through e-mail to obtain the missing information. All authors agreed upon the final selection of included studies.

The inclusion criteria were as follows: (i) observational studies (cohort or registry), (ii) provision of endpoint for overall survival or all-cause mortality in malignancy patients aged 0-39 years with different health insurance status, and (iii) report of effect estimates: hazard ratio (HR), relative risk (RR), or Odds ratios (OR) and available relevant raw data for recalculation. 
The exclusion criteria were: (i) case report, comment, editorial, letter, quasi-experiment (non-random subject assignment), or unpublished study and (ii) abstract or conference proceeding. Of two or more articles from the same team or organization, only the latest publication or the report with the largest sample size was selected.

\section{Data isolation and quality assessment}

Two investigators independently extracted all information of interest in a standardized form, including the study design, name of first author, title, country, publication year, follow-up duration, endpoints, sample size, adjustment level, mean age, gender, analysis strategy (statistical models and adjustment factors), and effect magnitude, including HRs, RRs, or ORs, as well as relevant raw data for re-calculation.

Study quality was evaluated by two independent investigators using the Effective Public Health Practice Project Quality Assessment Tool for Quantitative Studies. ${ }^{17}$ Any inconsistencies were addressed via discussions. This quality assessment tool rates the study procedures as 'strong', 'moderate', or 'weak' using eight scales (selection bias, study design, confounders, blinding, data collection methods, withdrawal/ dropouts, intervention integrity, and analyses). We scored a paper as overall 'strong' or 'high quality' if no 'weak' item score existed and if at least four of the eight items were 'strong'. A paper of overall 'moderate quality' was one with only one 'weak' item score, with otherwise only 'strong' and 'moderate' item scores. The remaining studies were rated as 'weak' or 'low quality' overall.

\section{Statistical analyses}

The primary outcome measure was overall survival (freedom from all-cause mortality) ${ }^{18}$ or all-cause mortality. Dichotomous outcomes were synthesized using HRs, RRs, or ORs, with 95\% confidence interval (CIs). The percent of between-study variability due to between-study heterogeneity was estimated using $\mathrm{I}^{2}$ statistic ${ }^{19}$ and was classified as high, modest, and low with $\mathrm{I}^{2} \geq 50 \%,<50 \%$, and $<25 \%$, respectively. A CI for $\mathrm{I}^{2}$ was identified using the iterative non-central chi-square method. ${ }^{20}$ RRs were converted to natural logarithms, and logRRs and standard errors were pooled by DerSimonian and Laird's approach in a random- or fixed-effect model as per the heterogeneity evaluated by Cochran's $Q$ and $\mathrm{I}^{2}$ statistics.

One-study-removed analysis was also performed to test sensitivity. Regarding the a-priori discrepancy of all-cause mortality, we conducted subgroup analyses by age (0-21 years, 15-39 years, and 0-39 years) according to the patient population of the enrolled studies. Publication bias was quantified by Egger's test (regression asymmetry) and Begg's test (rank correlation). All analyses were conducted using Stata statistical 15.0 (Stata Corp LP), at the $\alpha$ level of 0.05. P was set as two-sided. The 95\% CI with null ' 1 ' indicated no clinical significance even if $\mathrm{P}<0.05$.

\section{Results}

\section{Study selection, characteristics, and quality} assessment

The initial search found 3867 potentially feasible articles, and after title and abstract screening, 52 articles were retrieved for full-text assessment. Finally, 14 studies $^{11,14-16,21-30}$ were included (Fig. 1). Of the 14 studies, the dates of publication were between 2009 and 2019, the sample size varied between 19 and 80,855 patients, and there were four resources. Four studies were conducted among children, six among AYAs and four included both groups. Patient demographics, tumor characteristics, and treatments are shown in Table I.

Eleven of the 14 identified programs were assessed as moderate via the global rating, three as weak, and none as strong (Table II). Study design and confounders were the main weaknesses. 


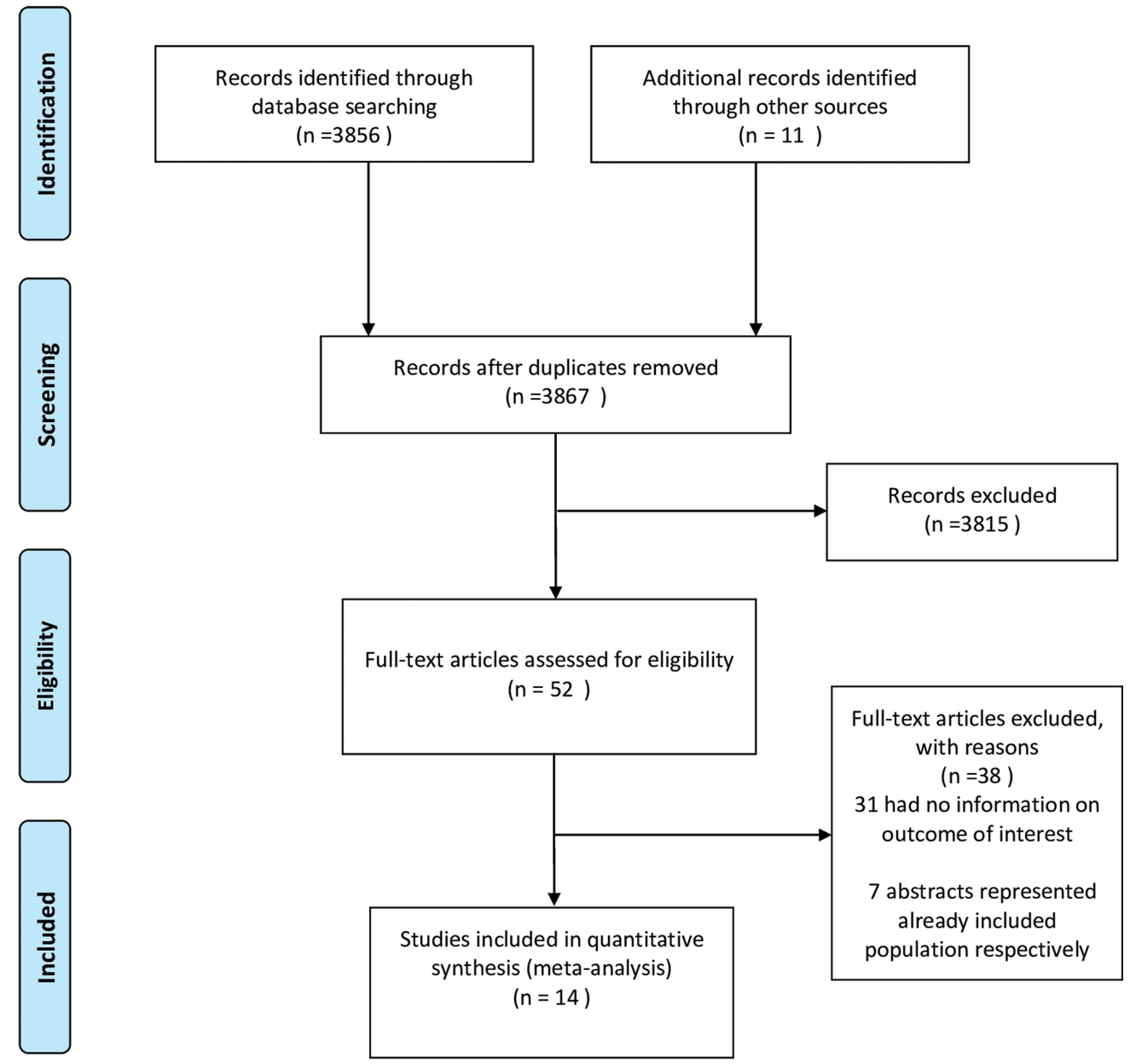

Fig. 1. Flow-diagram of study selection for the systematic literature review.

Effect of health insurance status on all-cause mortality in malignancy in patients aged 0-39 years

In 10 studies which examined the relationship between insured and uninsured patients, the pooled RR of all-cause mortality for insured versus uninsured was 0.78 (95\% CI, 0.71-0.86; $\mathrm{p}<0.001)$ in a fixed-effects model without heterogeneity $\left(I^{2}=33.7 \%, p=0.138\right.$; Fig. 2). A stratified analysis by contingency revealed the presence of positive outcome regarding all-cause mortality in studies among children and AYAs (HR 0.76, 95\% CI 0.68-0.84; $\mathrm{p}<0.001$; Fig. 3). Since Egger's test showed evidence of publication bias ( $p=0.012$, Fig. 4), rather than replacing potential missing data, we performed a trim-and-fill sensitivity analysis and found basically similar results.
In 10 studies reporting the relationship between private and nonprivate insurance, the pooled RR of all-cause mortality for private insurance versus nonprivate insurance was $0.70 \quad(95 \%$ CI 0.60 to $0.82 ; \mathrm{p}<0.001$ ) in a random-effect model with severe heterogeneity $\left(\mathrm{I}^{2}=83.3 \%\right.$, $\mathrm{p}$ $<0.001$; Fig. 5). Heterogeneity was analyzed via the sensitivity test. However, heterogeneity remained after the exclusion of single studies. The funnel plots showed evidence of systematic bias in the analysis of all-cause mortality (Begg test, $\mathrm{p}=0.21$; Egger's test, $\mathrm{p}=0.005$; Fig. 6). In the exploration of possible publication bias via the trim-and-fill approach, we did not substitute the probable missing data and found generally identical results. 


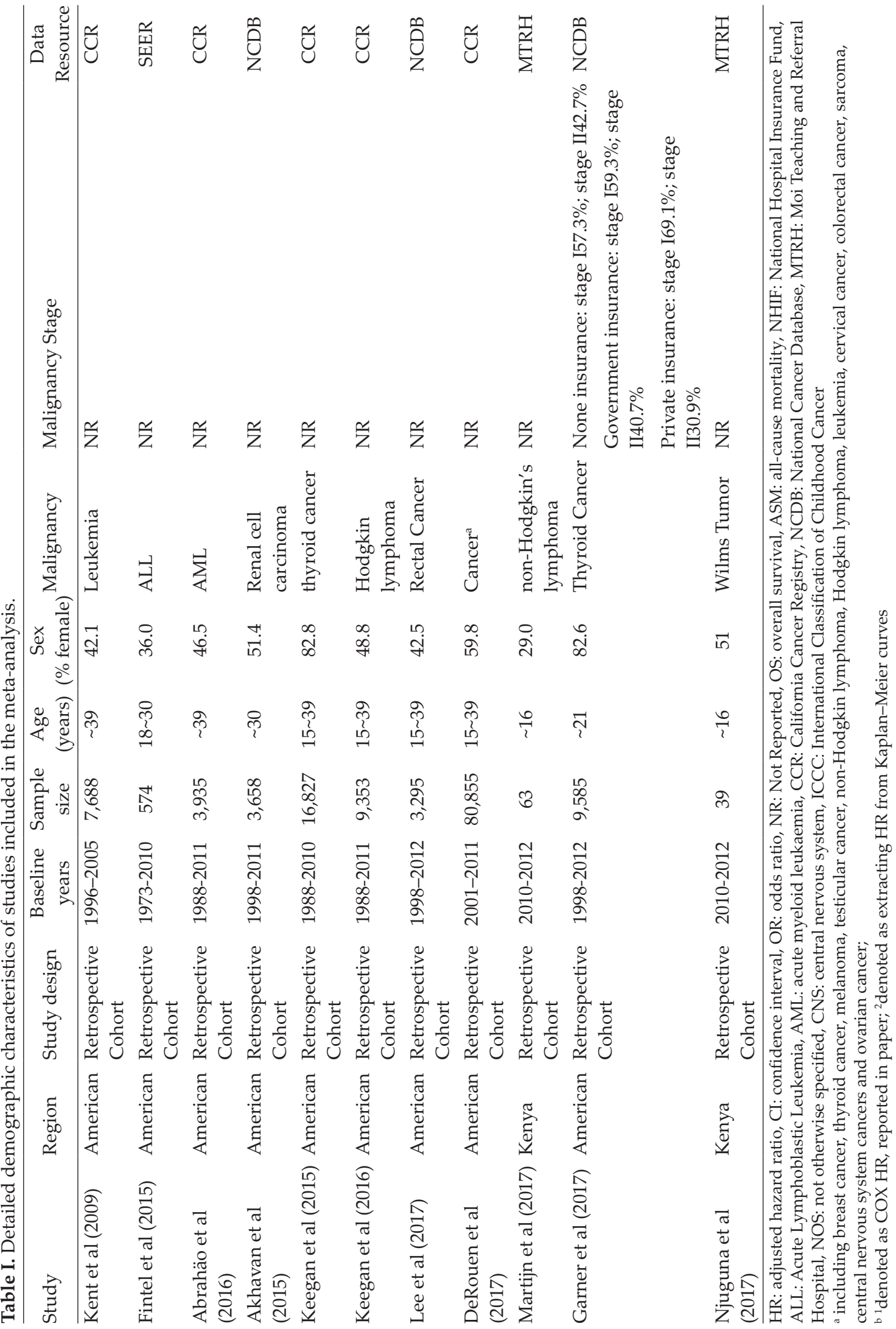




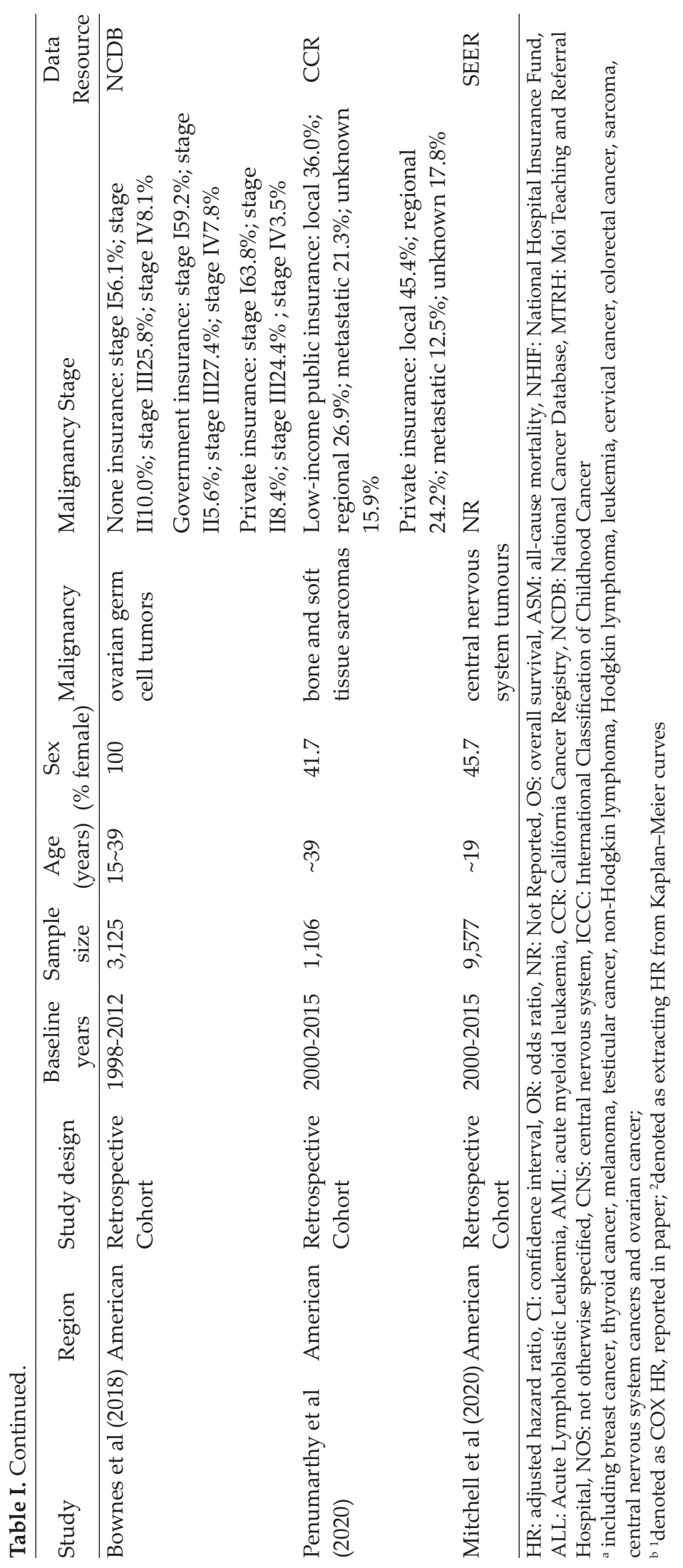




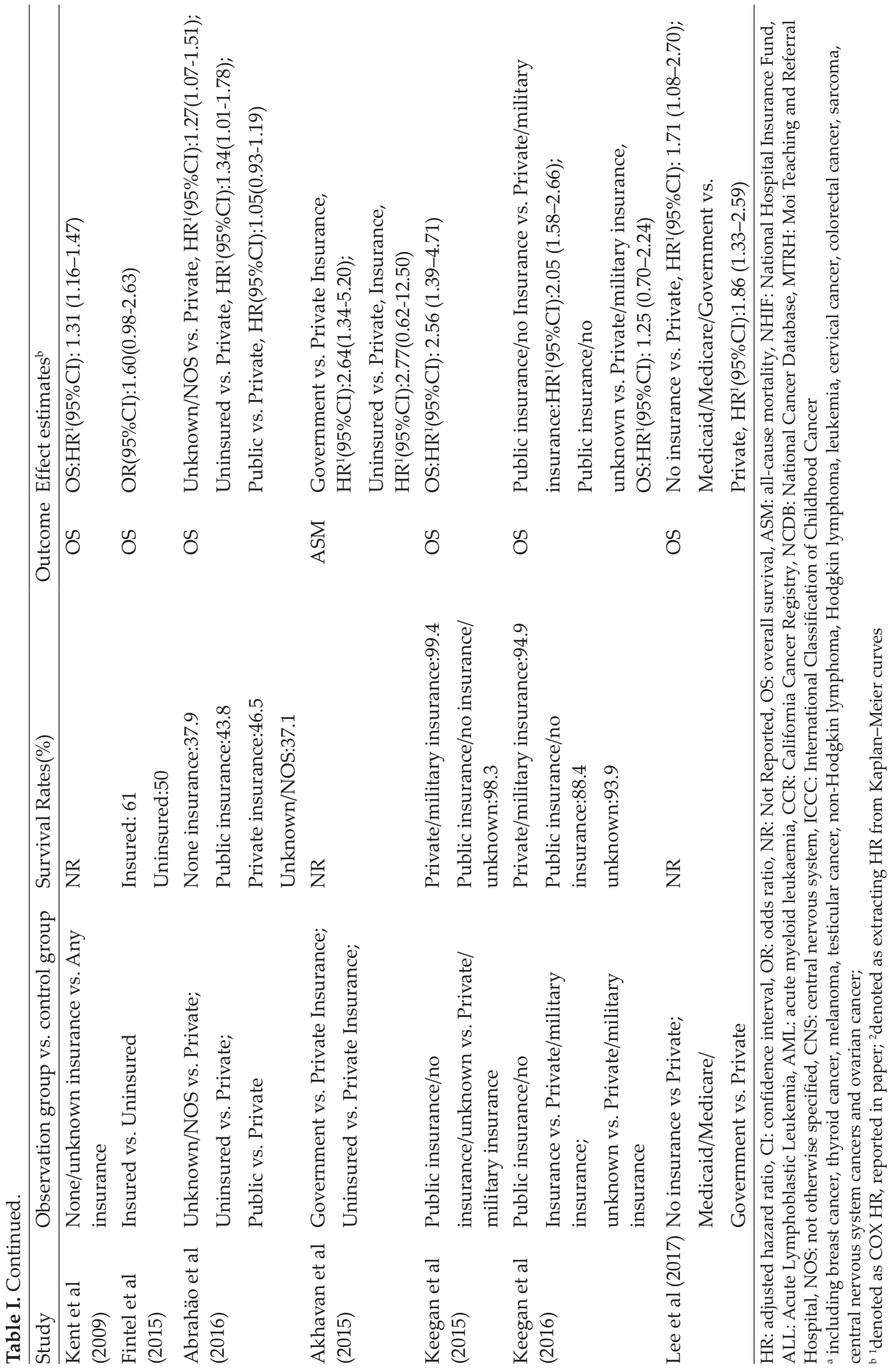




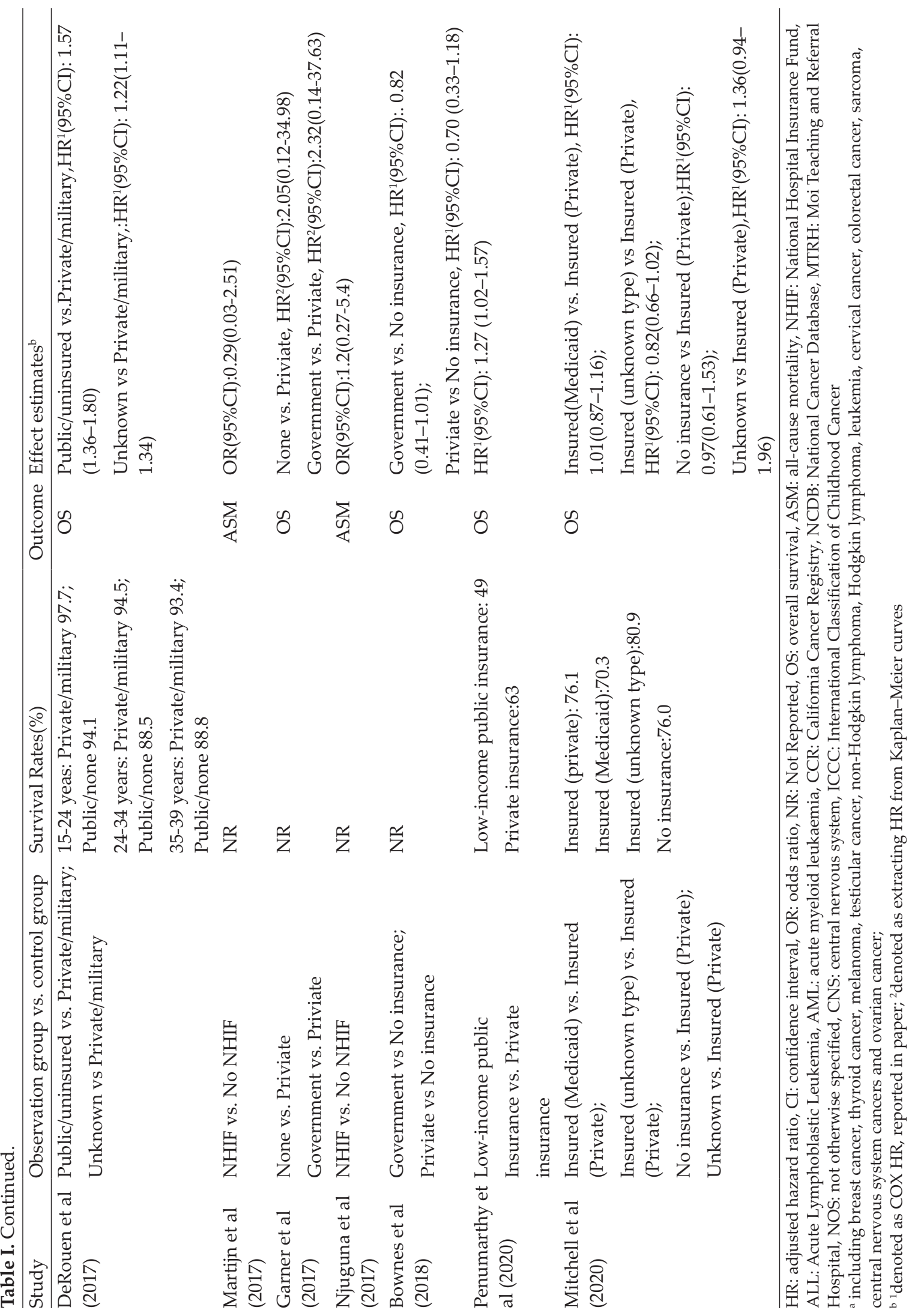




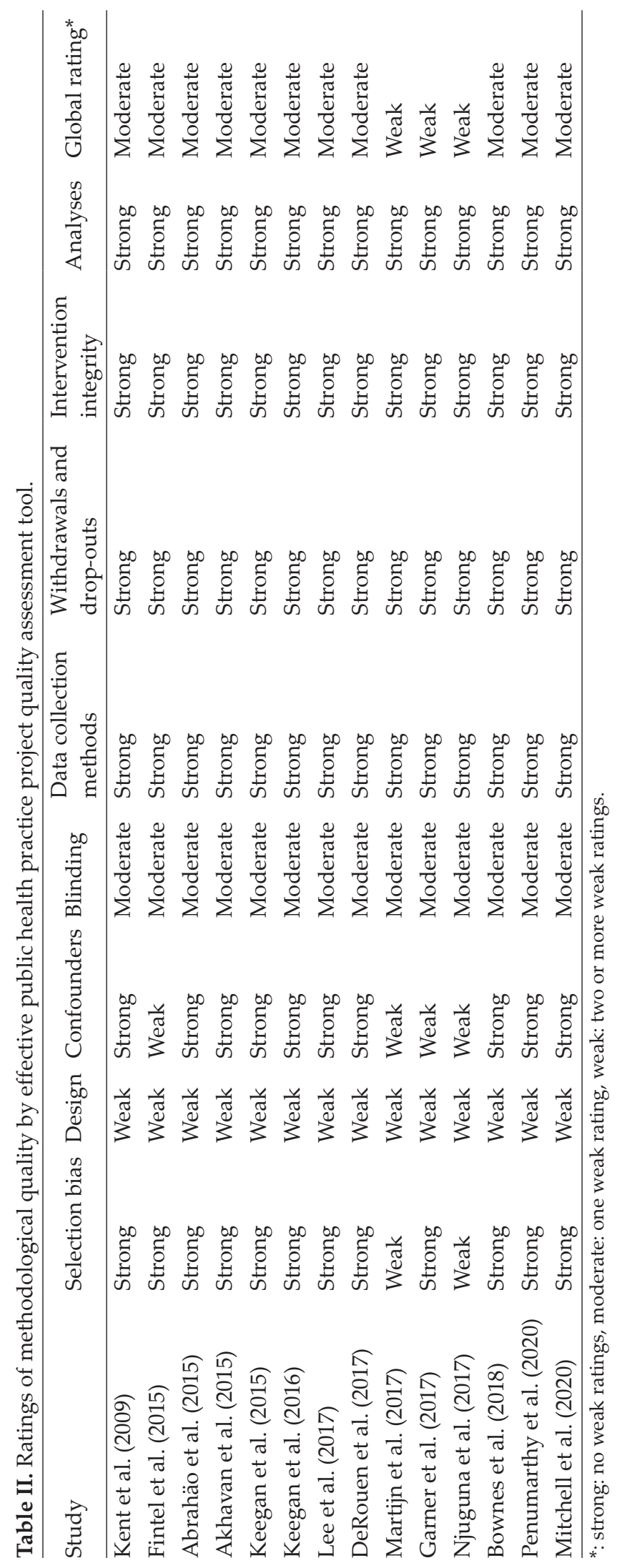




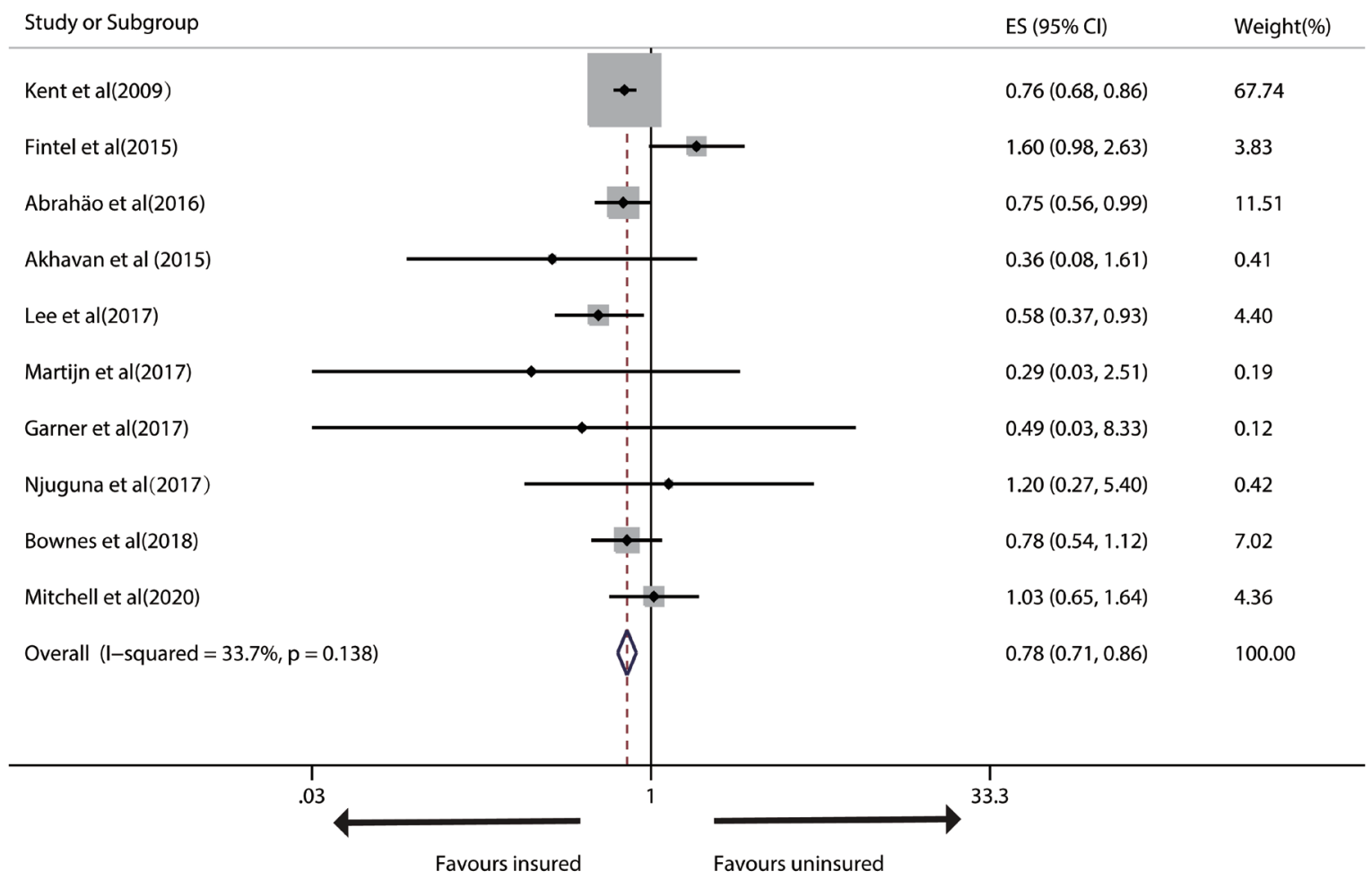

Fig. 2. Forest plot of the risk of all-cause mortality between insured and uninsured cancer patients aged 0-39 years.

\section{Discussion}

This systematic review found that uninsured malignancy patients, aged $0-39$ years, relative to private insurance, had a higher risk of all-cause mortality. Many factors (such as disease stage and delayed diagnosis) related to healthcare availability may have modulated the association between deficient or public insurance and survival, ${ }^{31}$ which in turn resulted in a later stage diagnosis (and resulting in the cancer being untreatable), delays, lower therapy reception, termination, ${ }^{13}$ Medicaid service inhibitions, or limited accessibility of information and support services among patients and survivors. ${ }^{32}$ The AYA Health Outcomes and Patient Experience research found that insurance shortage was related to a lower quality of life among new patients. ${ }^{33}$ Similarly, contacts with health care services may promote adherence to therapy and follow-up instructions, which are factors that were reportedly connected with poor outcomes among AYAs.
However, the race and SES differences in survival identified among privately insured AYAs imply that broadening insurance coverage alone may not eliminate all outcome differences. Insurance coverage has often been shown to be a key modulator of race and SES differences in survival. This is supported by recent studies, including ours, on relationships between no or public insurance and cancer outcomes among AYAs after race and SES adjustments. ${ }^{34}$ Our results verified the significance of insurance coverage, and suggested that considerable race and SES disparities still exist even among those with private insurance. Our results agree with recent findings that severe financial stress is related to a cancer diagnosis for those who are relatively young in age, lower SES, or nonwhite race/ethnicity, irrespective of insurance conditions. ${ }^{35}$ For those with private insurance, financial stress can originate from out-of-pocket costs related to co-payments, co-insurance, deductibles, and out-of-network costs, that may dictate treatment plans and termination, and 


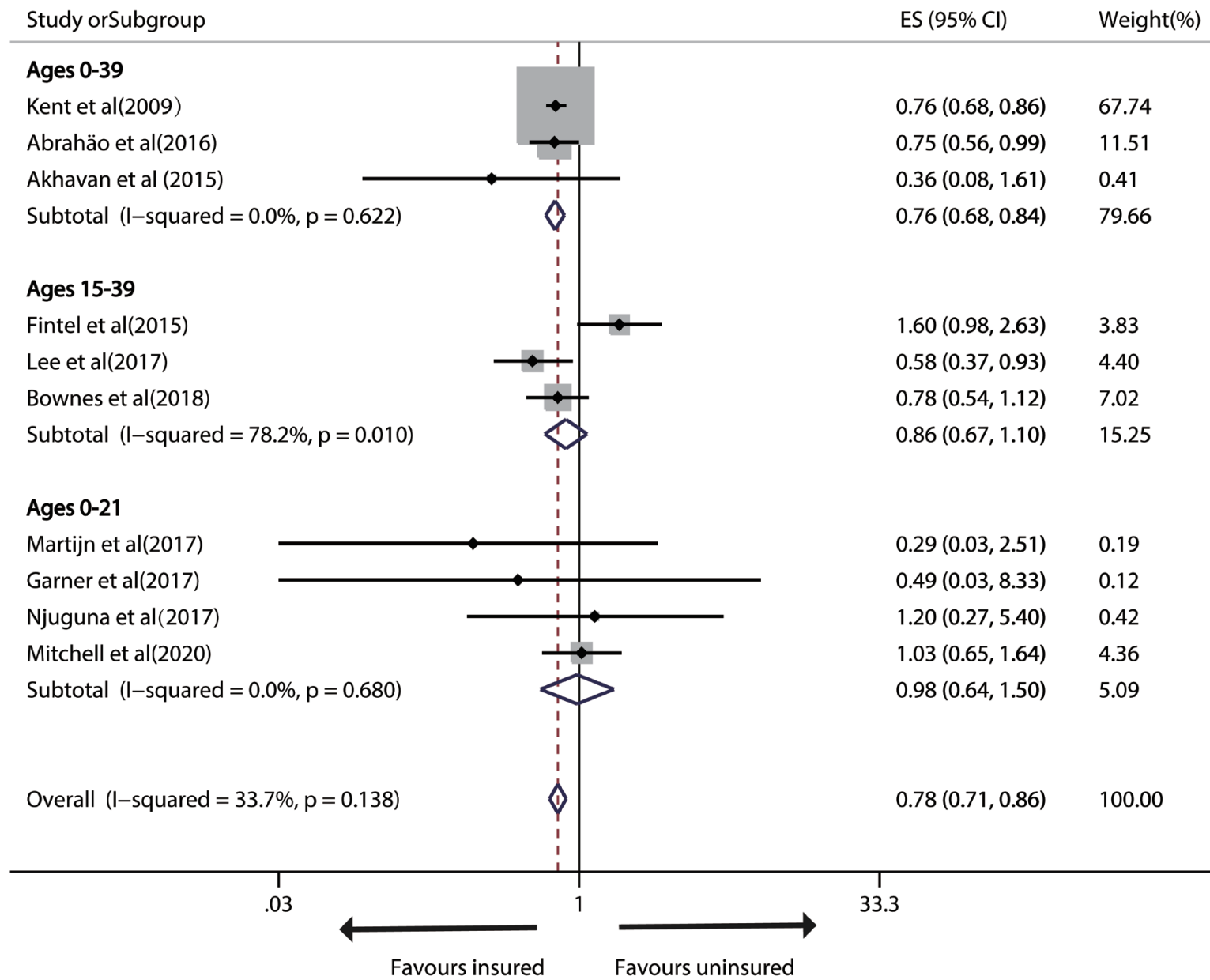

Fig. 3. Forest plot of the risk of all-cause mortality between insured and uninsured cancer patients aged 0-39 years, according to study population.

general well-being. ${ }^{36}$ For instance, for all age groups, African Americans and low-SES citizens are less likely to undergo standard medical therapy than their Caucasian counterparts, even when they have the same insurance coverage. This disparity has been noted by other studies on specific cancer care. ${ }^{37}$

Race bias may affect care use (regardless of insurance condition), and chronic burden due to bias affects health outcomes. ${ }^{38}$ Severe racial/ ethnic differences for cancers resulting in some groups being more prone to avoidance of therapy indicate that disparities in socioeconomic resources (social capital) may contribute to and intensify the residual racial/ethnic disparities in cancer survival. ${ }^{39}$ Financial concerns among minorities and low-SES groups, even with private insurance, may critically contribute to racial/ethnic and SES differences in therapy and therefore, survival. Moreover, biological divergences in cancer subtypes are probably related to some cancers. For example, AfricanAmerican AYAs are more susceptible to breast cancer of specific molecular subtypes, and these are related to more adverse prognoses..$^{40} \mathrm{In}$ addition, when considering molecular subtypes and insurance types in survival models, these decrease the relationship of a certain race/ ethnicity with a severe risk of death. ${ }^{41}$

Our analyses have some notable limitations. First, we did not examine the roles of specific sociodemographic characteristics (e.g., income, education, and access to medical care). There may also have been some undetected 


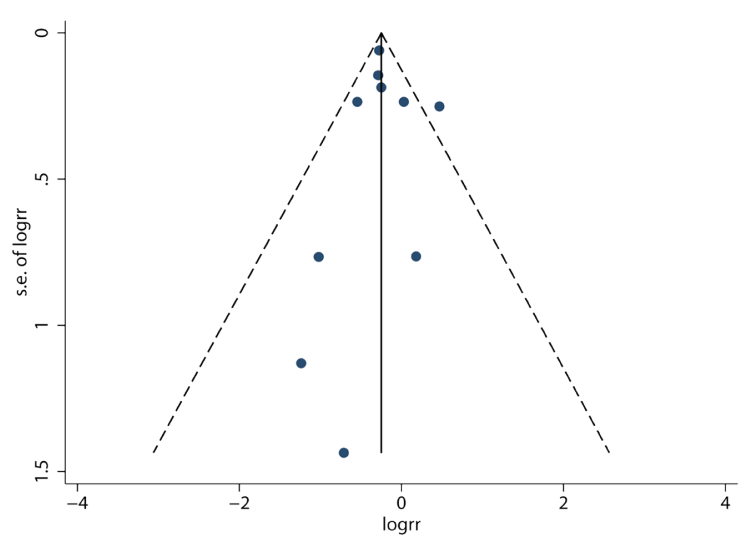

Fig. 4. Funnel plot of the risk of all-cause mortality between insured and uninsured cancer patients aged 0-39 years.

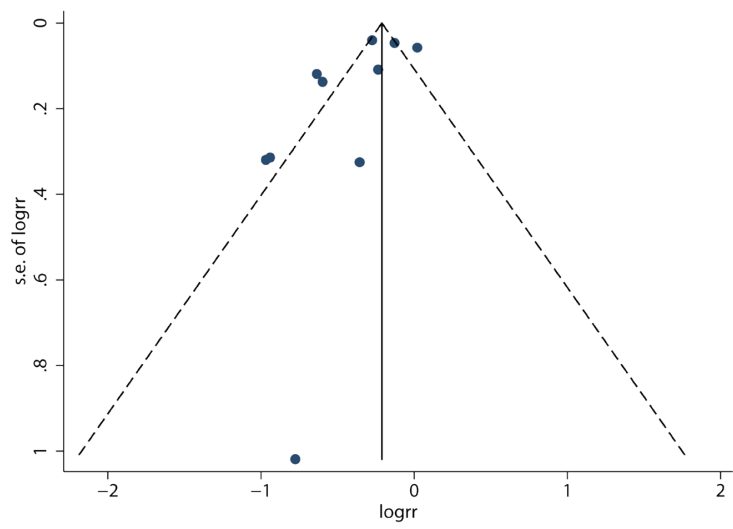

Fig. 6. Funnel plot of the risk of all-cause mortality between private and nonprivate insurance cancer patients aged 0-39 years.

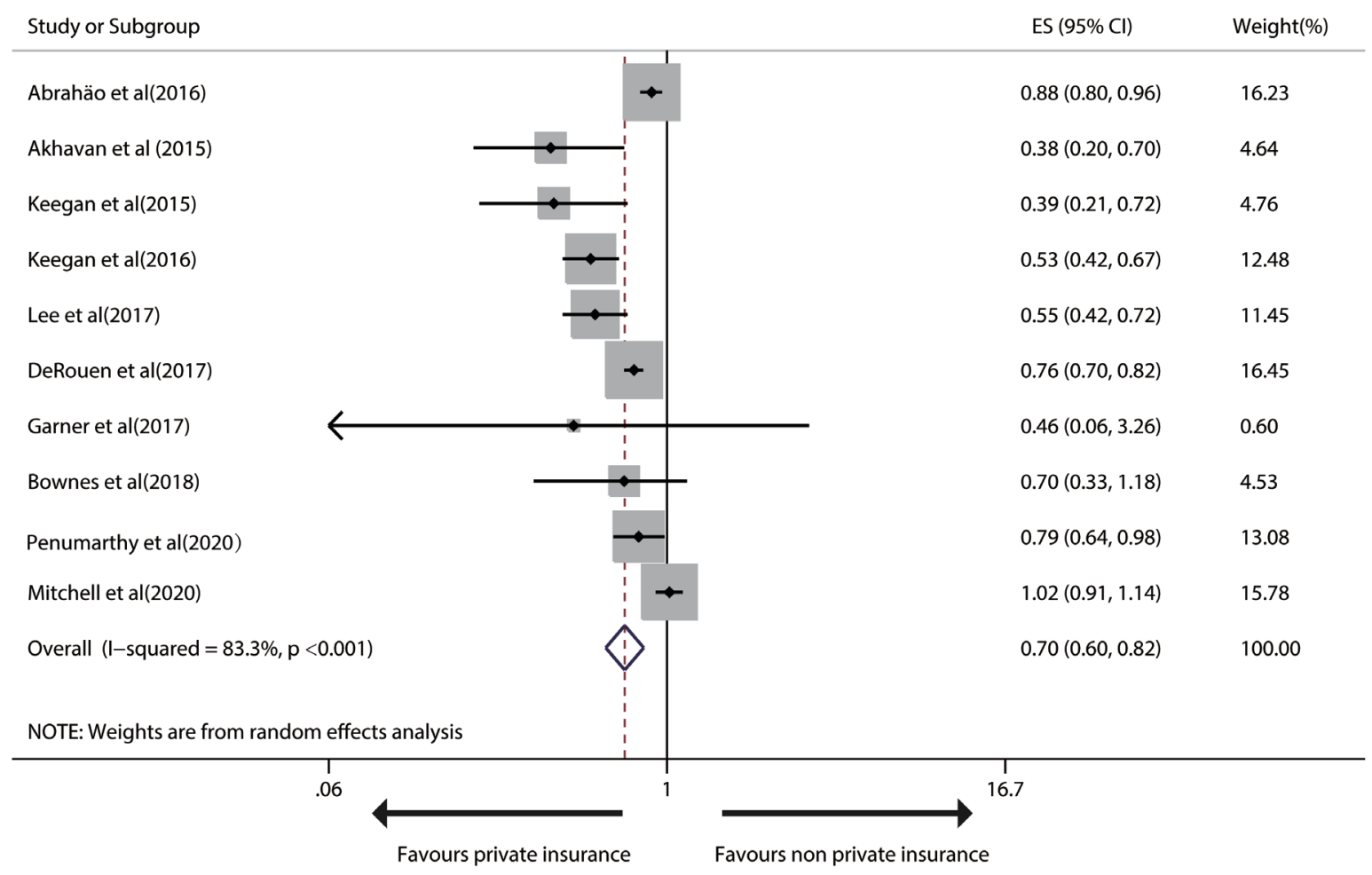

Fig. 5. Forest plot of the risk of all-cause mortality between private and nonprivate insurance cancer patients aged 0-39 years.

confounding factors that we did not account for in our findings. Second, we ignored therapeutic indices, which were often inconsistent and/or incomplete. This omission is likely to weaken our findings because we cannot clarify the extent to which the divergences in survival may be induced by differences in treatment (which are also probably related to insurance coverage). Third, we were unable to detect causality in a cross-sectional analysis and there were uncertainties regarding whether deficient or private insurance may lead to delayed diagnoses. Fourth, since insurance status was reported at diagnosis, we cannot explain the 
changes in insurance status over the follow-up period. As a result, uninsured patients may be misclassified as Medicaid patients at baseline, which may lead to a lower observed survival rate among Medicaid patients..$^{42}$ We are not inferring that Medicaid is less 'protective' than private insurance. Rather, our concern is that any shortage of insurance upon diagnosis may be related to a higher risk and that patients coded as 'Medicaid-insured' may actually be uninsured before diagnosis. Fifth, any survival superiority for privately insured patients may be ascribed to lead-time bias, which was suggested as a reason for survival differences by insurance conditions among adults. ${ }^{42}$ For instance, the lead-time of later diagnoses may give a false impression of longer survivals among privately insured patients than among uninsured or Medicaid patients. In addition, as anticipated in any systematic review, the cohort studies demonstrated remarkable heterogeneity for all-cause mortality between uninsured and privately insured patients. Moreover, Egger's test uncovered a potential publication bias, which was difficult to identify. These results suggest that we may have exaggerated the exact effect if some studies, such as abstracts or conference proceedings being a potential resource for grey literatures were excluded.

In conclusion, results from our systematic review suggest that limited or deficient insurance is heavily related to all-cause mortality in AYAs with malignancy. Strategies aimed at identifying causality and reducing disparities are warranted.

\section{Acknowledgements}

This research was supported by Shanghai Philosophy and Social Science Program (No.2018BSH002).

\section{Author contribution}

The authors confirm contribution to the paper as follows: study conception and design: $\mathrm{SH}, \mathrm{CH}$; data collection: $\mathrm{HH}$; analysis and interpretation of results: $\mathrm{CH}, \mathrm{LJ}$; draft manuscript preparation: $\mathrm{CH}, \mathrm{HL}$. All authors reviewed the results and approved the final version of the manuscript.

\section{Conflict of interest}

The authors declare that they have no competing interest.

\section{Supplementary information is available at:}

http://www.turkishjournalpediatrics.org/ uploads/turkjped.2021.04.001.S1.pdf

\section{REFERENCES}

1. Pritchard-Jones $\mathrm{K}$, Pieters R, Reaman GH, et al. Sustaining innovation and improvement in the treatment of childhood cancer: lessons from highincome countries. Lancet Oncol 2013; 14: e95-e103.

2. Bleyer A. The adolescent and young adult gap in cancer care and outcome. Curr Probl Pediatr Adolesc Health Care 2005; 35: 182-217.

3. Global Burden of Disease Child and Adolescent Health Collaboration, Kassebaum N, Kyu HH, Zoeckler $\mathrm{L}_{\text {, }}$ et al.Child and adolescent health from 1990 to 2015: findings from the global burden of diseases, injuries, and risk factors 2015 Study. JAMA Pediatr 2017; 171: 573-592.

4. Patton GC, Coffey C, Sawyer SM, et al. Global patterns of mortality in young people: a systematic analysis of population health data. Lancet 2009; 374: 881-892.

5. Abdul-Razak S, Azzopardi PS, Patton GC, Mokdad $\mathrm{AH}$, Sawyer SM. Child and adolescent mortality across Malaysia's epidemiological transition: a systematic analysis of global burden of disease data. J Adolesc Health 2017; 61: 424-433.

6. Heron M. Deaths: leading causes for 2010. Natl Vital Stat Rep 2013; 62: 1-96.

7. Sidebotham P, Fraser J, Fleming P, Ward-Platt M, Hain R. Patterns of child death in England and wales. Lancet 2014; 384: 904-914.

8. Bleyer A, Ferrari A, Whelan J, Barr RD. Global assessment of cancer incidence and survival in adolescents and young adults. Pediatr Blood Cancer 2017; 64 .

9. Mogensen H, Modig K, Tettamanti G, Erdmann F, Heyman M, Feychting M. Survival after childhood cancer-social inequalities in high-income countries. Front Oncol 2018; 8: 485. 
10. Stones DK, De Bruin GP, Esterhuizen TM, Stefan DC. Childhood cancer survival rates in two South African units. S Afr Med J 2014; 104: 501-504.

11. Keegan TH, Grogan RH, Parsons HM, et al. Sociodemographic disparities in differentiated thyroid cancer survival among adolescents and young adults in California. Thyroid 2015; 25: 635648 .

12. James SL, Castle CD, Dingels ZV, et al. Global injury morbidity and mortality from 1990 to 2017: results from the Global Burden of Disease Study 2017. Inj Prev 2020; 26(Suppl 1): i165.

13. Aizer AA, Falit B, Mendu ML, et al. Cancer-specific outcomes among young adults without health insurance. J Clin Oncol 2014; 32: 2025-2030.

14. Keegan TH, DeRouen MC, Parsons HM, et al. Impact of treatment and insurance on socioeconomic disparities in survival after adolescent and young adult Hodgkin lymphoma: a population-based study. Cancer Epidemiol Biomarkers Prev 2016; 25 : 264-273.

15. Fintel AE, Jamy O, Martin MG. Influence of insurance and marital status on outcomes of adolescents and young adults with acute lymphoblastic leukemia. Clin Lymphoma Myeloma Leuk 2015; 15: 364-367.

16. Kent EE, Sender LS, Largent JA, Anton-Culver H. Leukemia survival in children, adolescents, and young adults: influence of socioeconomic status and other demographic factors. Cancer Causes Control2009; 20: 1409-1420.

17. Docrat S, Besada D, Cleary S, Lund C. The impact of social, national and community-based health insurance on health care utilization for mental, neurological and substance-use disorders in lowand middle-income countries: a systematic review. Health Econ Rev 2020; 10: 11.

18. Takagi H, Umemoto T; ALICE (All-Literature Investigation of Cardiovascular Evidence) Group. Worse survival after transcatheter aortic valve implantation than surgical aortic valve replacement: a meta-analysis of observational studies with a propensity-score analysis. Int J Cardiol 2016; 220: 320-327.

19. Higgins JP, Thompson SG. Quantifying heterogeneity in a meta-analysis. Stat Med 2002; 21: 1539-1558.

20. Hedges LV, Pigott TD. The power of statistical tests in meta-analysis. Psychol Methods 2001; 6: 203-217.

21. Abrahão R, Keogh RH, Lichtensztajn DY, et al. Predictors of early death and survival among children, adolescents and young adults with acute myeloid leukaemia in California, 1988-2011: a population-based study. Br J Haematol 2016; 173: 292-302.
22. Akhavan A, Richards M, Shnorhavorian M, Goldin A, Gow K, Merguerian PA. Renal cell carcinoma in children, adolescents and young adults: a National Cancer Database study. J Urol 2015; 193: 1336-1341.

23. Lee DY, Teng A, Pedersen RC, et al. Racial and socioeconomic treatment disparities in adolescents and young adults with stage II-III rectal cancer. Ann Surg Oncol 2017; 24: 311-318.

24. DeRouen MC, Parsons HM, Kent EE, Pollock BH, Keegan THM. Sociodemographic disparities in survival for adolescents and young adults with cancer differ by health insurance status. Cancer Causes Control 2017; 28: 841-851.

25. Martijn HA, Njuguna F, Olbara G, et al. Influence of health insurance status on paediatric non-Hodgkin's lymphoma treatment in Kenya. BMJ Paediatr Open 2017; 1: e000149.

26. Garner EF, Maizlin II, Dellinger MB, et al. Effects of socioeconomic status on children with welldifferentiated thyroid cancer. Surgery 2017; 162: 662-669.

27. Njuguna F, Martijn HA, Kuremu RT, et al. Wilms tumor treatment outcomes: perspectives from a lowincome setting. J Glob Oncol 2017; 3: 555-562.

28. Bownes LV, Stafman LL, Maizlin II, et al. Socioeconomic disparities affect survival in malignant ovarian germ cell tumors in AYA population. J Surg Res 2018; 222: 180-186.e3.

29. Penumarthy NL, Goldsby RE, Shiboski SC, Wustrack R, Murphy $\mathrm{P}$, Winestone LE. Insurance impacts survival for children, adolescents, and young adults with bone and soft tissue sarcomas. Cancer Med 2020; 9: 951-958.

30. Mitchell HK, Morris M, Ellis L, Abrahão R, Bonaventure A. Racial/ethnic and socioeconomic survival disparities for children and adolescents with central nervous system tumours in the United States, 2000-2015. Cancer Epidemiol 2020; 64: 101644.

31. Smith EC, Ziogas A, Anton-Culver H. Delay in surgical treatment and survival after breast cancer diagnosis in young women by race/ethnicity. JAMA Surg2013; 148: 516-523.

32. Keegan TH, Tao L, DeRouen MC, et al; AYA HOPE Study Collaborative Group. Medical care in adolescents and young adult cancer survivors: what are the biggest access-related barriers? J Cancer Surviv 2014; 8: 282-292.

33. Smith AW, Bellizzi KM, Keegan TH, et al. Healthrelated quality of life of adolescent and young adult patients with cancer in the United States: the adolescent and young adult health outcomes and patient experience study. J Clin Oncol 2013; 31: 21362145. 
34. Gorey KM, Luginaah IN, Holowaty EJ, Zou G, Hamm C, Balagurusamy MK. Mediation of the effects of living in extremely poor neighborhoods by health insurance: breast cancer care and survival in California, 1996 to 2011. Int J Equity Health 2013; 12: 6.

35. Yabroff KR, Dowling EC, Guy GP Jr, et al. Financial hardship associated with cancer in the United States: findings from a population-based sample of adult cancer survivors. J Clin Oncol 2016; 34: 259-267.

36. Zafar SY, Peppercorn JM, Schrag D, et al. The financial toxicity of cancer treatment: a pilot study assessing out-of-pocket expenses and the insured cancer patient's experience. Oncologist 2013; 18: 381-390.

37. Wu XC, Lund MJ, Kimmick GG, et al. Influence of race, insurance, socioeconomic status, and hospital type on receipt of guideline-concordant adjuvant systemic therapy for locoregional breast cancers. J Clin Oncol 2012; 30: 142-150.
38. Chae DH, Nuru-Jeter AM, Adler NE, et al Discrimination, racial bias, and telomere length in African-American men. Am J Prev Med 2014; 46: 103-111.

39. Tehranifar P, Neugut AI, Phelan JC, et al. Medical advances and racial/ethnic disparities in cancer survival. Cancer Epidemiol Biomarkers Prev 2009; 18: 2701-2708.

40. Keegan TH, DeRouen MC, Press DJ, Kurian AW, Clarke CA. Occurrence of breast cancer subtypes in adolescent and young adult women. Breast Cancer Res 2012; 14: R55.

41. Keegan TH, Press DJ, Tao L, et al. Impact of breast cancer subtypes on 3-year survival among adolescent and young adult women. Breast Cancer Res 2013; 15: R95.

42. Bradley CJ, Gardiner J, Given CW, Roberts C. Cancer, medicaid enrollment, and survival disparities. Cancer 2005; 103: 1712-1718. 